\title{
Focused liver ablation by cavitation in the rabbit: a potential new method of extracorporeal treatment
}

\author{
F Prat, J-Y Chapelon, F Abou El Fadil, A Sibille, Y Theillière, T Ponchon, D Cathignol
}

\begin{abstract}
A new device was used to achieve focused tissue ablation by shockwave induced cavitation. The device produced a half cycle of negative pressure followed by a shock wave, thus enhancing cavitation. Twenty eight New Zealand rabbits were treated. Therapeutic ultrasound was targeted at the centre of the liver under ultrasound guidance. The focal volume was scanned with a computer operated $x-y-z$ micropositioner. The number and frequency of bursts as well as the distance between two $x-y-z$ displacements were preselected. The relation of tissue ablation seen to preselected parameters, effects on surrounding tissues, biological side effects, and mode of healing were studied. Macroscopy, planimetry, and quantitative microscopy were used. Focused and homogeneous tissue ablation was achieved within well defined limits. Maximal tissue ablation was seen in the centre of the target. Liver surrounding the target remained unaffected. Lesions were made of a-cellular spots surrounded by disorganised rims of necrotic hepatocytes; 24 hours after treatment, the changes (mean (SEM)) in alanine transaminase and haemoglobin were $+225(36) \%$ and $-2.4(2) \%$ respectively. Serum transaminases, haemoglobinaemia, and packed cell volume were normal 21 days after treatment and the target area was replaced by a fibrous scar. It is concluded that ultrasound cavitation may achieve extracorporeal intrahepatic tissue ablation inside a predetermined target. This technique should now be tested in an animal hepatic tumour model.

(Gut 1994; 35: 395-400)
\end{abstract}

Malignant tumours of the liver remain an important health problem throughout the world.' Non-surgical treatments have shown essentially palliative effects and surgery is potentially curative in only a small percentage of patients. ${ }^{2-4}$ Surgery needs the resection of nontumoral liver tissue and does not prevent recurrence. Recurrences can now be detected at early stages with the help of modern diagnostic methods. ${ }^{5}$ Hence, it seems reasonable to contemplate a minimally invasive method providing radical and selective cancer destruction, which could be applied to such tumours with some benefit. It was our hypothesis that acoustic cavitation induced by shock waves could be such a method. Cavitation is defined as the non-linear oscillation and collapse of gas bubbles suspended in a liquid under the influence of acoustic (mechanical) pressure waves ${ }^{6-10}$; the expansion of bubble nuclei results from a depression within the medium and their collapse may be induced by a sudden compression wave after expansion." It has been shown that cavitation, when occurring at an interface near a cell membrane for example, was deleterious to biological media through mechanical, non-thermal effects. ${ }^{12-18}$ We showed the feasibility of radical tissue destruction in the rabbit liver in vivo by a concomitant administration of focused extracorporeal electrohydraulic shock waves with intravascularly infused gas microbubbles. ${ }^{19}$ Our results showed improvement shortcomings, however, which made the method inapplicable to the human or even to animal models of tumours without an unacceptable toxicity: tissue ablation was not focused, non-homogeneous, and needed an invasive and potentially toxic intra-arterial infusion of gas microbubbles to be efficient. Our objectives were thus to perfect the method to enhance cavitation, eradicate bubble injection, and to improve the focusing and selectiveness of tissue destruction. In this study, qualitative and quantitative assessments of liver ablation in the rabbit show that improvements of the method have made these achievements possible.

\section{Materials and methods}

ANIMALS

Twenty eight New Zealand hybrid rabbits of both sexes (Elevage Scientifique des Dombes, Ain, France) weighing $2 \cdot 0$ to $2 \cdot 25 \mathrm{~kg}$ were used. All the experiments were conducted with premedication by intramuscular acepromazine (Vetoquinol, Lure, France) $4 \mathrm{mg} / \mathrm{kg}$ and induction and maintenance of anaesthesia by a continuous venous infusion of a mixture of ketamine (Substantia Parke-Davis, Courbevoie, France) $125 \mu \mathrm{g} / \mathrm{kg} / \mathrm{mn}$ and $2 \%$ xylazine (Bayer, Leverkusen, Germany) $1 \mu \mathrm{g} / \mathrm{kg} / \mathrm{mn}$. The abdomen was shaved thoroughly and the abdominal wall was covered with a coupling agent. The animals were positioned on a purpose built plexiglas set up.

\section{CAVITATION GENERATOR}

The generator included an IBM/PC compatible computer for the overall control of the system, a pulse generator, an ultrasonic pulser (spark gap), a three axis stepper motor controller, a stepper motor driven micropositioner arrangement, an ultrasound scanner used to visualise the target, and a tank filled with degassed and deionized water (Fig 1A). The piezoelectric transducer array used as a shock wave source generator contained 18 single transducers mounted in a $28 \mathrm{~cm}$ diameter bowl and focused at $16 \mathrm{~cm}$. The transducers were mechanically adjusted to pro- 
Figure 1: (A) Arrangement of the device. (1) The parallelepipedic target inside (2) the liver; (3) the plexiglas set up can be moved in the $x, y$, and $z$ directions; the transducer and the animal are immersed into (4) degassed water; (5) imaging and targeting ultrasound $(U S)$ probe; $(B)$ pressure tracing of the generator, with an initial half cycle of depression (expansion wave) followed by a half cycle of peak positive pressure (shockwave)

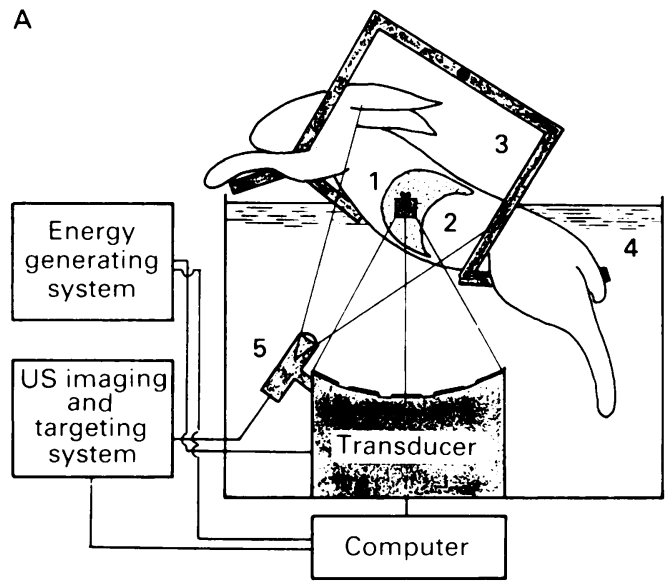

two $5 \mathrm{~mm}$ thick layers, whose length and width are multiples of the focal spot diameter. Each session was defined by the burst frequency and the distance between two points inside the target. The complete shock wave administration to the target was obtained through step by step shifts over the generator with the help of the $x-y-z$ micropositioner. As the creation of cavitation bubbles may oppose the penetration of acoustic waves and thus prevent formation of further lesions, the deepest layer was always treated first and the irradiation progressed from depth to surface, under ultrasound monitoring. The session stopped automatically at completion.

\section{METHODS}

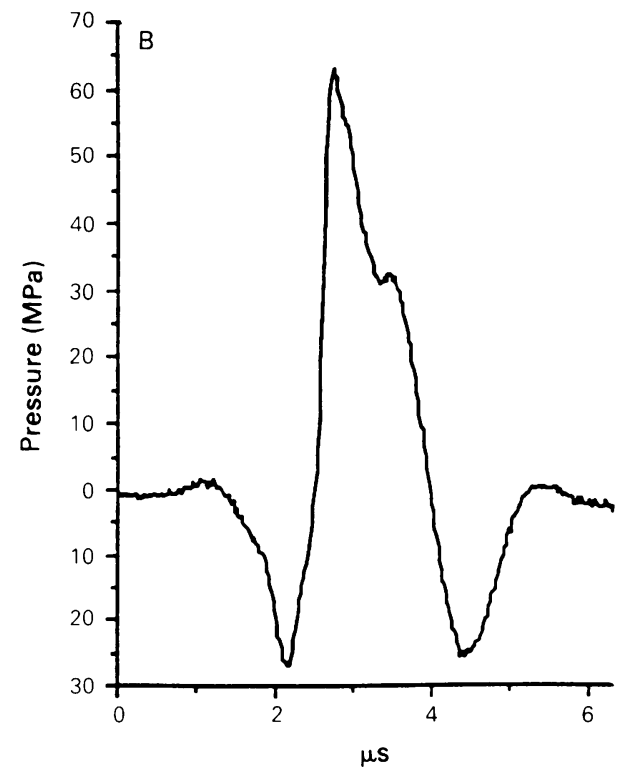

\section{Short term end points}

Macroscopy - after fixation of the liver in acetified formalin, the whole organ was cut in $5 \mathrm{~mm}$ thick sagittal slices; slices including necrosis were compared with a video print of a sagittal ultrasound scan taken during the session. This methodology was used to accurately assess the $\mathrm{x}, \mathrm{y}$, and $\mathrm{z}$ dimensions of the macroscopically affected zone (as measured twice with a calliper square); calculations were corrected to take into account the volume reduction subsequent to formalin fixation $(\times 0.96)$ and correlated with the predetermined target volume.

Light microscopy $-4 \mu \mathrm{m}$ thick tissue slices were prepared and stained with haemalum, eosin, and saffron (HES) for standard histological analysis.

Quantitative automated microscopy - this technique was used for the assessment of tissue lysis and viability: three $4 \mu \mathrm{m}$ thick sections from the centre of the target area of each specimen were stained with periodic acid Schiff (PAS); each section was analysed three times under $\times 10$ magnification (nine fields/analysis) on a Quantimet 570 image analyser (Cambridge, $\mathrm{UK}$ ); the analyser was requested to measure the surface of a section unstained with PAS (thus considered non-viable, even if morphologically unaffected) as a percentage of the whole surface included in the target area.

Planimetry was used to assess quality of tissue destruction as a function of the depth of the target. A video print was used as above to perform sectioning of the liver orthogonal to the $\mathrm{z}$ axis. Each $1 \mathrm{~mm}$ thick section was cut in $4 \mu \mathrm{m}$ thick slices and stained with HES. Stained slices were photographed and magnified; planimetry (Digital polar planimeter 330, Germany) was repeated three times on each section to measure the surface of the lytic areas as a percentage of the whole surface.

The anaesthesised animal was positioned on the plexiglas set up and the abdomen was immersed into the water bath; a sagittal image of the liver was obtained from the $3.5 \mathrm{MHz}$ scanning probe (Aloka, Japan) visualising the focus of the generator. With the help of the $x-y-z$ micropositioning system, the animal was moved over the generator to preposition the target over the focus. The target was defined as follows: located in the median lobe of the liver, top of the volume $25 \mathrm{~mm}$ deep from the abdominal wall, $16(\mathrm{x}) \times 16$ $(\mathrm{y}) \times 10(\mathrm{z}) \mathrm{mm}$ in overall dimensions - that is,

\section{Mid term end points}

Clinical parameters - general condition of the animal during and after treatment, progress, and recovery from anaesthesia, weight variation between treatment and death were noted.

Biological parameters - haemoglobinaemia, packed cell volume, and serum transaminases (alanine transaminase and aspartate transaminase) were assessed before treatment, 24 hours 
Figure 2: (A) Ultrasound sagittal scan of a rabbit liver during shockwave administration (top: abdominal wall; left $=$ heart cavities; bottom right $=$ stomach; centre=liver): the small echogenic spot inside the target area in the liver (arrow) is suggestive of cavitation bubble formation; (B) ultrasound sagittal scan of a rabbit liver at the end of a session; note the large hyperechogenic spot in the focal area (arrow).
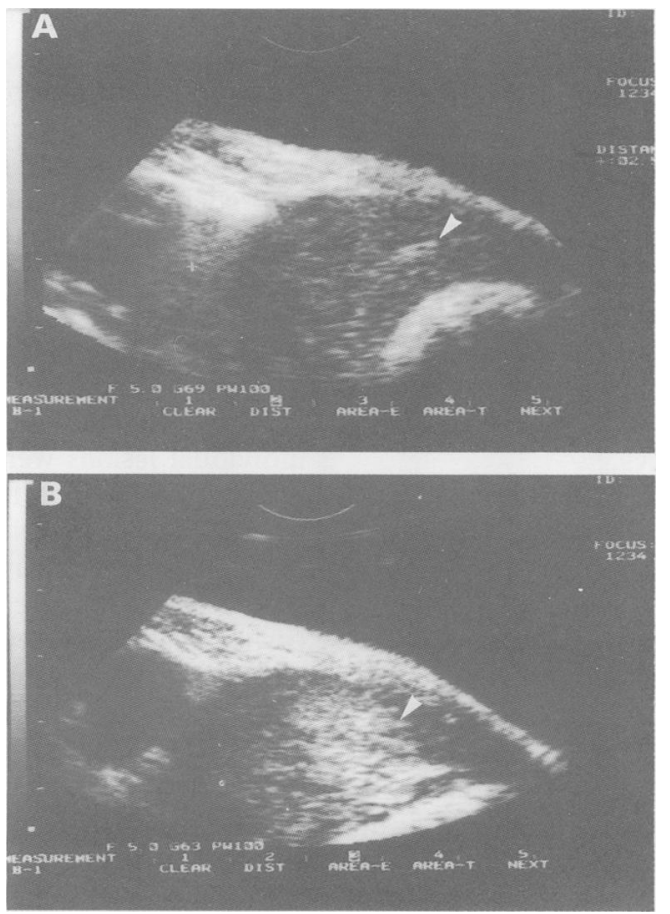

after, and 21 days after treatment in certain groups of animals.

Necropsy - thorough examination of the peritoneal cavity, diaphragm, lungs, heart, and kidneys was done. Particular attention was given to hepatic vessels damage.

TREATMENT PARAMETERS AND PROTOCOL

Twenty eight rabbits were assigned to the following groups of treatment:

Group 1 - eight rabbits were treated with the topographical parameters described above, at a burst frequency of $1 \mathrm{~Hz}$, with a distance between points of $2 \mathrm{~mm}$. They were killed 24 hours after treatment. Macroscopy of the liver, coeliac vessels, other intra-abdominal organs, kidneys, diaphragm, lungs, and heart was done, as well as

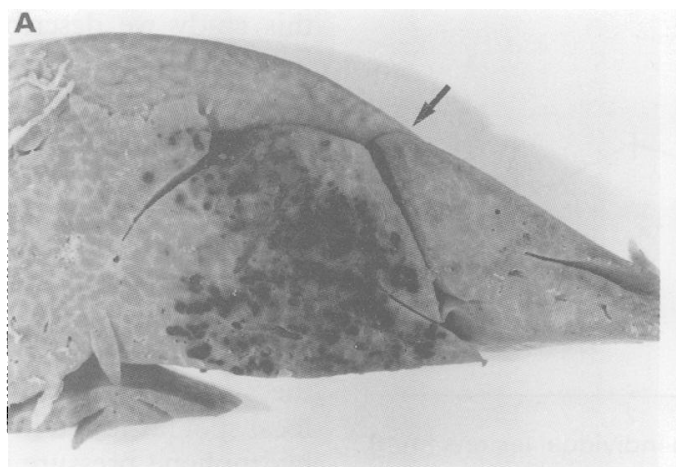

Figure 3: (A) Macroscopic view of a liver slice from the target zone (fixed in acetified formalin); the arrow shows the ultrasound beam incidence and shows that the superficial parenchyma is not affected; $(B)$ tissue ablation 24 hours after cavitation (PAS, original magnification $\times 2 \cdot 5)$. Bottom of the picture: central target zone; top of the picture: periphery of the target zone. A-cellular spots of necrosis are surrounded by a zone of PAS negative hepatocytes. Bottom right: note PAS negative area while lobular architecture remains visible; $(C)$ fibrous scar in the target area 21 days after cavitation Masson's trichrome, original magnification $\times 10$ ),

regeneration nodules present images of mitoses. a light microscopic examination of the target area, including quantitative microscopy.

Group 2 - eight rabbits treated with the same parameters were killed 21 days later; before death, all the animals were monitored clinically and biologically; on death, the animals had macroscopic and light microscopic examinations.

Group 3 - subgroups of three animals each were treated to assess the influence of the distance between two points $(1,2$, and $4 \mathrm{~mm})$ on the amount of tissue destruction, all other parameters remaining equal to group 1 . Twenty four hours after treatment, the target area was submitted to a quantitative microscopic analysis as described above.

Group 4 - three rabbits were treated with the same parameters as group 1; after death at 24 hours, the target area was submitted to a planimetric analysis of tissue destruction as a function of the depth from the anterior pole of the target area.

Group 5 - three rabbits were submitted to two consecutive treatment sessions, 48 hours distant from one another; targeting for the second session was based upon the hyperechogenic remnant from the first one; 24 hours after the second session, the target area was examined by light microscopy.

\section{Results}

ULTRASOUND MONITORING OF THE TREATMENT Progress of the treatment session was monitored through a sagittal view of the target zone, inside which tiny and fugitive hyperechogenic spots could be seen from the very first bursts; remnants of these spots gave the whole target area a whitish, brilliant aspect at the end of a session (Fig 2).

CLINICAL RESULTS

No animal died from anaesthesia during this

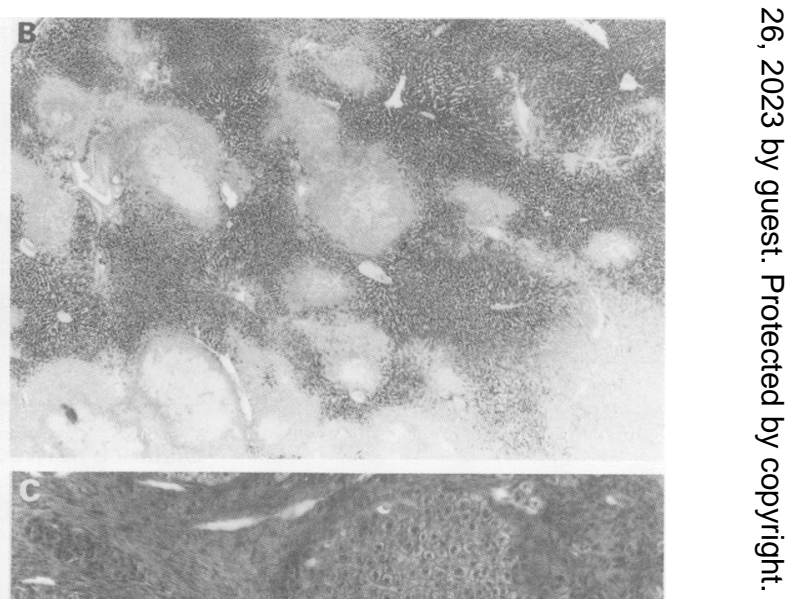


study; no animal died or presented any clinical complication of any sort during the follow up period, in any of the groups studied. Urine and faeces were normal in all cases. Food intake was unchanged after treatment and weight presented no abnormal (mean (SEM)) variation (2.17 $(0 \cdot 5) \mathrm{kg}$ after treatment compared with $2 \cdot 11(0 \cdot 3)$ before treatment). All animals presented petechiae $(0.5 \mathrm{~mm}$ in diameter $)$ over the abdominal wall, on a surface consistent with the cross section of the ultrasound beam at the skin level (diameter $2.5 \mathrm{~cm}$, section $4.9 \mathrm{~cm}^{2}$ ). Petechiae disappeared after a few days.

\section{BIOLOGY}

All animals from group 2 showed an increase in serum transaminase activities 24 hours after the session, between 1.5 and 6 times the normal value. Mean (SEM) variation of alanine transaminase was $+225(36) \%$; in all animals, the recovery was complete 21 days after treatment; haemoglobin records showed non-significant variations $(-2 \cdot 4(2) \%)$.

\section{HISTOLOGICAL EXAMINATION}

The target zone was always constituted of individual lytic spots surrounded by a rim of necrotic hepatocytes of disorganised architecture. The hepatic tissue around the affected area remained morphologically strictly normal (Fig 3A and B). Quantitative microscopy with PAS staining showed that tissue ablation was surrounded by a morphologically normal but unstained (non-viable) zone. In group 2, the livers examined 21 days after treatment presented always a fibrous whitish scar at the surface of the capsule; the scar was usually rectangular, $0.5 \mathrm{~cm}$ in width and less than $1 \mathrm{~cm}$ in length, retractile, with a regenerative aspect of the surrounding liver. The healing process was a collagenic fibrosis, poorly inflammatory, and sometimes nodular surrounded by proliferative hepatocytes presenting images of mitoses (Fig 3C).
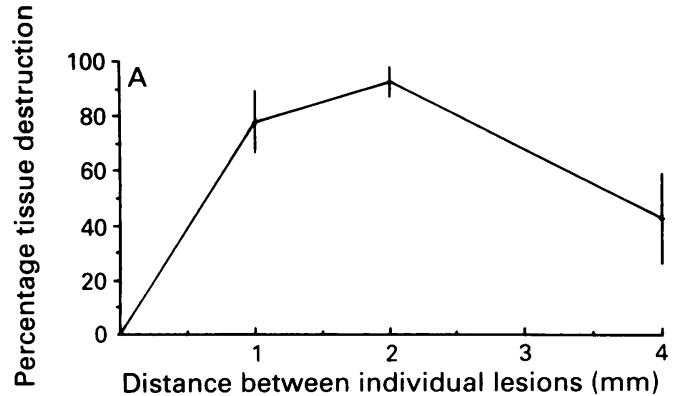

Figure 4: (A) Percentage of tissue ablation/section of the affected volume as a function of the distance between points as estimated by quantitative microscopy and colorimetry $(P A S) ;(B)$ percentage of tissue ablation as a function of the depth as a function of the depth
from the anterior pole of the target, as estimated by planimetry. Vertical bars represent standard error of the mean.

\section{ANALYSIS OF PARAMETERS}

Macroscopy - the distribution of affected tissue volumes from group 1 showed a dispersion around the predetermined (desired) volume; the mean (SEM) volume seen was $3.59(1.23) \mathrm{cm}^{3}$ for a predetermined volume of $2.56 \mathrm{~cm}^{3}$. The affected zone presented grossly more spheric than parallelepipedic, within sharp, well defined limits. Its position in the liver was always consistent with the desired coordinates - that is, in the centre of the organ and its top polar plane $25 \mathrm{~mm}$ deep from the skin. Within these limits, individual lesions were homogeneously distributed, although not falling into straight lines. Overall dimensions of the target zone of animals having two consecutive sessions (group 5) presented no difference with other groups, but tissue ablation presented was more complete, with almost no remaining viable hepatocytes.

The percentage of tissue affected (as assessed by quantitative microscopy) decreased significantly when the distance between points was increased from 2 to $4 \mathrm{~mm}(\mathrm{p}<0.01$, Student's $t$ test); however, a distance between points shorter than the focal spot diameter - that is, $1 \mathrm{~mm}$ - did not improve tissue ablation (Fig 4A).

The planimetric study of in depth tissue lysis (group 4) showed that the maximum effect was obtained $3 \mathrm{~mm}$ from the anterior (bottom) pole of the lesion ( $70 \%$ tissue lysis) and decreased anteriorly and posteriorly (Fig 4B).

\section{Discussion}

In a previous study, we have shown the feasibility of tissue destruction in the liver of rabbits by cavitation. ${ }^{19}$ Several shortcomings appeared, however, because of the technology used to produce cavitation: (1) the lesions obtained were poorly focused; (2) the need for an intravascular infusion of gas bubbles to enhance cavitation was invasive and difficult; (3) bubbles were not small enough to diffuse into liver capillaries and subsequently, bubbles trapped in the portal spaces generated mostly portal and periportal lesions, while the lobule was generally not affected. In this study we describe reproducible and well focused lesions in the liver by an extracorporeal approach, without the need to inject bubbles in the circulation. Toxicity was not serious; there were no deaths; biological side effects were constantly noted, but always transitory and with complete recovery after three weeks. There were no perforations of surrounding structures, intraabdominal haemorrhages or thromboses of large vessels. These improvements were permitted by the small size and constant positioning of the focal spot (as tested in vitro in our laboratory by hydrophone pressure recordings (data not provided), and mostly by the effect of the initial negative half cycle of the pressure tracing (expansion wave). High intensity ultrasound produces cytotoxicity through essentially hyperthermia and cavitation; heating is certainly the dominant mechanism of tissue destruction at intensities below $1000 \mathrm{~W} / \mathrm{cm}^{2}$ and exposure times longer than one second ${ }^{20}$; several research groups, as well as ours, are trying to develop devices for extracorporeal tumour treatment based on focused, high intensity necrosis induc- 
ing hyperthermia. ${ }^{20-23}$ In this study, it can be assumed that hyperthermia plays a very marginal part, if any, and that almost exclusively cavitation participates in the creation of tissue necrosis.

Cytotoxicity such as seen here is probably thus both direct, by changes of cell and nuclear membranes, cytoskeleton, and mitochondria ${ }^{24}$ and indirect through microvascular disruption ${ }^{6}$ and production of free radicals. ${ }^{25}$ It is very difficult to assess accurately the occurrence of cavitation in vivo ${ }^{26}$; ; however, the hyperechogenic spot induced by each burst at the focal point in the liver, as seen by ultrasound monitoring, is the landmark of transitory bubble production - that is, of cavitation.

Another advantage of this technology is the reproductiveness of the lesion in a given model. A perfect alignment of individual lesions, however, was not seen; this is partly because of physiology (respiration movements) and also because of acoustic phenomena of diffraction, refraction, and interactions with the blood flow, which are not well understood in living media. A discrepancy was noted between the desired volume of tissue necrosis and the lesion seen; this is partly explained by respiratory movements, because shots were not breath monitored; cavitation can also be created early ahead of the focus, thus extending the lesion beyond the desired volume. ${ }^{25}$ Testing the role of the distance between points in the target clearly showed that this parameter influenced the density of tissue ablation; the absence of increase in tissue ablation for a distance shorter than the focal spot diameter may be a result of cavitation bubbles occurring when a lesion was created very close to the next one ( $1 \mathrm{~mm}$ distant), thus preventing the creation of the next lesion. The actual amount of tissue ablation, however, included a rim of hepatocytes morphologically unchanged but functionally affected, as shown by quantitative microscopy with PAS staining.

Several other parameters might influence the quality of tissue ablation, which could not be investigated with this experimental model: in the liver for instance, the quality and quantity of lesions will be mostly influenced by the acoustic attenuation of the tissue as a function of depth. ${ }^{28}$ To study easily the conditions of tissue ablation deeper than $30 \mathrm{~mm}$ from the skin would need bigger animal models. Modulations of the amplitude of the acoustic wave (its negative as well as its positive components), of the peak positive and negative pressures or of the burst frequency might prove important for this factor. The histological lesion induced in the liver by cavitation is a necrosis entailing both microvascular lesions and direct parenchymal cell lysis. In contrast with our initial study, tissue ablation here affected the lobule as well as the portal space. The lesions were sharply defined, and the surrounding tissue seemed unaffected. The tissue effect was immediate and radical, and seemed to generate a repair process stimulating both collagenic fibrosis and hepatic regeneration. Indeed, tissue destruction is temporary and not all cells are killed by cavitation, suggesting that once applied to tumours, the remaining living cancer cells would continue growing. This important point can be considered in several ways: firstly, microvascular damage results in ischaemia and fibrosis; it can thus be expected that cells in the focal area continue to die after shockwave is given, and that tumour growth rate would seriously be affected, as has already been shown in vitro ${ }^{29}$ and in vivo. ${ }^{30}$ Secondly, the repetition of treatment sessions will probably complete and reinforce therapeutic effects. Thirdly, cavitation, as a local treatment, should probably be part of combined therapeutic schedules including chemotherapy or immunomodulators for instance. Several recent studies conducted by various laboratories have shown encouraging results. ${ }^{30-34}$

The development of a cavitation based device for the local treatment of liver cancer in humans is readily conceivable: it could be an alternative to surgery in certain cases of liver cancers. We are now investigating the effects of cavitation in a liver cancer model in the rabbit.

Preliminary elements of this study were presented as abstracts at the Journées Francophones de Pathologie Digestive, Paris, 1992 and at the First United Gastroenterology Week, Athens, 1992.

This work was supported in part by the Région Rhône-Alpes and an ARC grant, No 6833.

1 World Health Organization. Prevention of liver cancer: report of WHO meeting. In: World Health Organization Technical Report Series. Geneva: World Health Organization, 1983: 7-8.

2 Johnson PJ, Wilkinson ML, Karani J. Advances in neoplastic disease of the liver and biliary tract. Gut 1991; 32: S104-10.

3 Franco D, Capussotti L, Smadja C, Bouzari H, Grange D Dellepiane M. Resection of hepatocellular carcinoma; results in 72 European patients with cirrhosis. Gastro enterology 1990; 97: 733-8.

4 Holm A, Bradley E, Aldrete J. Hepatic resection of metastasis from colorectal carcinoma. Morbidity, mortality and pattern of recurrence. Ann Surg 1989; 209: 478-84.

5 Okuda K. Early recognition of hepatocellular carcinoma. Hepatology 1986; 6: 729-38.

6 Miller DL. A review of the ultrasonic bioeffects of microsonation, gas-body activation, and related cavitation-like phenomena. Ultrasound Med Biol 1987; 13: 443-70.

7 Flynn HG. Physics of acoustic cavitation in liquids. In: Mason WP, ed. Physical acoustics: principles and methods. Vol 1B. WP, ed. Physical acoustics: principles and

8 Dear JP, Field JE, Walton AJ. Gas compression and jet formation in cavities collapsed by a shock wave. Nature 1988; 332: 505-8.

9 Delius M, Muller M, Vogel A, Brendel W. Shock waves and cavitation. In: Ferrucci JT, Delius M, Burhenne HJ, eds. Biliary lithotripsy. Chicago: Year Book Medical Publishers, 1989: 23-8.

10 Coleman AJ, Saunders JE, Crum LA, Dyson M. Acoustic cavitation generated by an extracorporeal shockwave lithotripter. Ultrasound Med Biol 1987; 13:69-76.

11 Lauterborn W, Hentschel W. Cavitation bubble dynamics studied by high speed photography and holography: part 1 . Ultrasonics 1985;23: 260-8.

12 Ellwart JW, Brettel H, Kober LO. Cell membrane damage by ultrasound at different cell concentrations. Ultrasound Med Biol 1988; 14: 43-50.

13 Morton KI, ter Haar GR, Stratford IJ, Hill CR. The role of cavitation in the interaction of ultrasound with V79 chinese hamster cells in vitro. Brf Cancer 1982; 45: 147-50.

14 Ponchon T, Barkun A, Berger F, Ayela P, Margonari J, Capron E. Experimental tissue lesions related to extracorporeal lithotripsy of gallbladder. Surg Gynecol Obste 1989; 97: 457-63.

15 Sacks PG, Miller MW, Sutherland RM. Response of multicel spheroids to $1 \mathrm{MHz}$ ultrasonic irradiation: cavitation-related damage. Radiat Res 1983; 93: 545-59.

16 Vivino AA, Boraker DK, Miller D, Nyborg W. Stable cavitation at low ultrasonic intensities induces cell death and inhibits ${ }^{3} \mathrm{H}$-TdR incorporation by Con-A-stimulated murine inhibits ${ }^{3} \mathrm{H}$-TdR incorporation by Con-A-stimulated murine

lymphocytes in vitro. Ultrasound Med Biol 1985; 11: 751-9.
17 Chen You Lung, Israelachvili J. New mechanism of cavitation damage. Science 1991; 252: 1157-60.

18 Flint EB, Suslick KS. The temperature of cavitation. Science 1991; 253: 1397-9.

19 Prat F, Ponchon T, Berger F, Chapelon JY, Cathignol D. Hepatic lesions in the rabbit induced by acoustic cavitation Gastroenterology 1991; 100: 1345-50.

20 Yang R, Reilly CR, Rescorla FJ, Faught PR, Sanghvi NT, Fry FJ, et al. High-intensity focused ultrasound in the treatment of experimental liver cancer. Arch Surg 1991; 126: 1002-10.

21 ter Haar G, Sinnett D, Rivens I. High intensity focused ultrasound - a surgical technique for the treatment of discrete liver tumours. Phys Med Biol 1989; 34: 1743-50. 
22 Chapelon JY, Margonari J, Vernier F, Gorry F, Ecochard R, Gelet A. In vivo effects of high intensity ultrasound on Gelet A. In vivo effects of high intensity ultrasound on prostatic adenocarcir

23 Frizzell LA, Linke CA, Carstensen EL, Fridd CW. Thresholds for ultrasonic lesions in rabbit kidney, liver and testicle. IEEE Trans Biomed Eng 1977; 24: 393-6.

24 Steinbach $P$, Hofstädter F, Nicolai H, Rössler W, Wieland W. In vitro investigations on cellular damage induced by high energy shock waves. Ultrasound Med Biol 1992; 18: 691-2. Colend hiolo , Saunders JE. A review of the physical properties used in extracorporeal lithotripsy. Ultrasonics 1993; 31: 7589.

26 Edmonds PD, Ross P. Acoustic emission as a measure of exposure of suspended cells in vitro. Ultrasound Med Biol 1986; 12: 297-305.

27 Williams AR, Delius M, Miller DL, Schwarze W. Investigation of cavitation in flowing media by lithotripter shock waves both in vitro and in vivo. Ultrasound Med Biol 1989; 15: 53-60.

28 Fry FJ, Reilly CR, Dines KA, Etchison MR, Trauner EJ. Absorption in liver at the focus of an ultrasonic shock wave field. Ultrasound Med Biol 1991; 17: 65-9.
29 Prat F, Chapelon JY, Chauffert B, Ponchon T, Cathignol D. Cytotoxic effects of acoustic cavitation on HT-29 cells and a rat colon carcinomatosis in vitro. Cancer Res 1991; 51: $3024-9$.

30 Prat F, Chapelon JY, Abou El Fadil F, Theillière Y, Ponchon $T$, Cathignol $D$. In vivo effects of cavitation alone or in combination with chemotherapy in a peritoneal carcinomatosis in the rat. $\mathrm{Br} \mathcal{F}$ Cancer 1993; 68: 13-7.

31 Gambihler S, Delius M, Brendel W. Biological effects of shock waves: cell disruption, viability, and proliferation of L1210 cells exposed to shock waves in vitro. Ultrasound Med Biol 1990; 16: 587-94.

32 Russo P, Stephenson RA, Mies C, Huryk R, Heston WDW, Melamed MR, et al. High energy shock waves suppress
tumor growth in vitro and in vivo. $\mathcal{F}$ Urol 1986; 135: tumor

33 Weiss N, Delius M, Gambihler S, Dirschedl P, Goetz A, Brendel W. Influence of the shock wave application mode on the growth of A-Mel 3 and SSK2 tumors in vivo. Ultrasound Med Biol 1990; 16: 595-605.

34 Warlters A, Morris DL, Cameron-Strange A, Lynch W. Effect of electro-hydraulic and extracorporeal shock waves on gastrointestinal cancer cells and their response to cytotoxic agents. Gut 1992; 33: 791-3. 